\title{
17 STIMULATION OF BONE RESORPTION IN CALVARIAL BONES BY TOLL-LIKE2 RECEPTOR THROUGH ENHANCED RANKL
}

Ali kassem, ${ }^{1}$ Pedro C C SOUZA, ${ }^{1,2}$ Pernilla Lundberg, ${ }^{1}$ Catharina Lindholm, ${ }^{3}$ Ulf H Lerner1,3 ${ }^{1}$ Molecular Periodontology, Umeå University, Umeå, Sweden; ${ }^{2}$ Department of Physiology and Pathology, University of São Paulo State, Araraquara, Brazil, ${ }^{3}$ Centre for Bone and Arthritis Research at Institute for Medicine, Sahlgrenska Academy at University of Gothenburg, Gothenburg, Sweden

10.1136/annrheumdis-2011-201237.17

Background Bone loss in inflammatory diseases like periodontitis, rheumatoid arthritis, septic arthritis and loosened joint prosthesis or tooth implants is being considered a consequence of cytokine induced RANKL and subsequent enhanced osteoclast formation. During the last decade it has been recognised that a variety of cells express receptors (pathogenrecognition receptors $=\mathrm{PRP}$ ) for specific signatures of different pathogens (pathogen-associated molecular patterns PAMP) and endogenous stress signals (danger-associated molecular patterns DAMP), including Toll-like receptors (TLR).

Material and methods/results The authors used organ cultured neonatal mouse calvarial bones and isolated periosteal osteoblasts which express TLRs to study the role of TLR2 in bone resorption. LPS from the perio-pathogenic bacterium Porphyromonas gingivalis $\left(\mathrm{Pg}_{\mathrm{g}}\right.$ which is a weak agonist for TLR4 but a strong for TLR2 because of the contaminating lipoprotein), enhanced number of osteoclasts, ${ }^{45} \mathrm{Ca}$ release and bone matrix degradation (CTX) by a process inhibited by osteoprotegerin and zolendronic acid. LPS Pg enhanced the expression of osteoclastic genes (c-Fos, trap, oscar and cathepsin K) and reduced the expression of osteoblastic genes (osteocalcin, runx2, alp and procollagen $\alpha 1$ ). The effects were associated with increased mRNA and protein expression of RANKL, whereas OPG mRNA and protein were unaffected. Similar to LPS $P g, \mathrm{Pam}_{2} \mathrm{CSK}_{4}$ (synthetic ligand for TLR2/TLR6), $\mathrm{Pam3CSK}_{4}$ (ligand for TLR1/TLR2), HKLM (a heat killed preparation of Listeria monocytogenes, a TLR2 agonist) and FSL1 (a synthetic lipoprotein representing the N-terminal part of the 44-kDa lipoprotein LP44 of Mycoplasma salivarium) stimulated ${ }^{45} \mathrm{Ca}$ release and increased the mRNA expession of cathepsin $\mathrm{K}$ and RANKL, without affecting OPG. Pam2 also increased CTX release and RANKL-protein expression without affecting that of OPG. All TLR agonists increased the mRNA expression of IL-1 $\beta$, TNF $\alpha$, IL- 6 and COX-2. LPS P.g. and Pam 2 enhanced the $\mathrm{PGE}_{2}$ release from the bones. IL-1 $\beta$ and TNF $\alpha$ enhanced RANKL mRNA to a similar extent as LPS $P g$ and PGE $_{2}$ caused a $50 \%$ response, whereas IL- 6 had no effect. Anti-IL-1 $\beta$ and anti-TNF $\alpha$ did not affect LPS $P g$ induced RANKL mRNA. In the presence of an inhibitor of prostaglandin biosynthesis, the response to LPS Pg was reduced by $50 \%$ but still causing a considerable enhancement of RANKL mRNA. Stimulation of isolated calvarial osteoblasts with all TLR agonists increased RANKL mRNA expression, without affecting OPG mRNA.

Conclusion These data show that stimulation of TLR 2 results in bone resorption mediated by increased RANKL in osteoblasts and may be one mechanism for developing inflammatory bone loss. 